\title{
Imaging of prolonged BOLD response in the somatosensory cortex of the rat
}

\author{
Sarah Sonnay ${ }^{a}$, Nathalie Just ${ }^{b *}$, João M. N. Duarte ${ }^{a}$ and Rolf Gruetter ${ }^{a, c, d}$
}

\begin{abstract}
Blood oxygenation level-dependent (BOLD) functional MRI is a widely employed methodology in experimental and clinical neuroscience, although its nature is not fully understood. To gain insights into BOLD mechanisms and take advantage of the new functional methods, it is of interest to investigate prolonged paradigms of activation suitable for long experimental protocols and to observe any long-term modifications induced by these functional challenges. While different types of sustained stimulation paradigm have been explored in human studies, the BOLD response is typically limited to a few minutes in animal models, due to fatigue, anesthesia effects and physiological instability. In the present study, the rat forepaw was electrically stimulated for $\mathbf{2} \mathbf{h}$, which resulted in a prolonged and localized cortical BOLD response over that period. The stimulation paradigm, including an inter-stimulus interval (ISI) of $10 \mathrm{~s}$, that is $\mathbf{2 5 \%}$ of the total time, was applied at constant or variable frequency over $\mathbf{2} \mathbf{h}$. The steady-state level of the BOLD response was reached after 15-20 min of stimulation and was maintained until the end of the stimulation. On average, no substantial loss in activated volume was observed at the end of the stimulation, but less variability in the fraction of remaining activated volume and higher steady-state BOLD amplitude were observed when stimulation frequency was varied between 2 and $3 \mathrm{~Hz}$ every $5 \mathrm{~min}$. We conclude that the combination of ISI and variable stimulus frequency reproducibly results in robust, prolonged and localized BOLD activation. Copyright $\odot 2015$ John Wiley \& Sons, Ltd.
\end{abstract}

Keywords: BOLD; fMRl; electrical; forepaw; prolonged stimulation; animal model

\section{INTRODUCTION}

Blood oxygenation level-dependent (BOLD) functional MRI (fMRI) is a widely employed methodology in both clinical settings (1-3) and preclinical studies (4-9). Specific brain activation has been demonstrated using a variety of short paradigms in normal and diseased brain, although the understanding of the nature of the BOLD signal is still incomplete. Recently, taking advantage of increased magnetic fields, several studies have given access to functional metabolic changes occurring during brain activity using functional MRS (6,10-12). These new functional methods may at some point help unravel the fundamental mechanisms behind BOLD signals.

If prolonged stimulation paradigms have been developed quite straightforwardly for human investigations $(11,12)$, it has been more challenging in rodents due to neuronal and hemodynamic adaptations, anesthesia and difficulties in maintaining physiological conditions $(6,10)$. Neuronal and hemodynamic adaptations are often perceived as a decrease of BOLD signal amplitude $(1-3,6)$. In the case of neuronal adaptation, this decline is notably due to adjustments of neuronal outputs to previous stimuli to better encode small stimulus modifications or to neuronal hyperpolarization without modification in synaptic inputs (13). Hemodynamic adaptation rather reflects a mismatch between cerebral metabolic rate of oxygen $\left(\mathrm{CMRO}_{2}\right)$ and cerebral blood flow (CBF) $(2,14)$. Anesthesia might affect the neurovascular coupling (15) by modifying baseline neuronal activity (16) and altering spontaneous action potentials. Anesthesia can also modulate the degree of neuronal adaptation and neuronal excitability during stimulation by acting on different molecular targets, such as potassium cation channels and N-methyl-Daspartate (NMDA) receptors (17), or gamma-aminobutyric acid type A receptors in the case of $\alpha$-chloralose (18). Hyper- and hypoventilation conditions not only affect blood oxygen content, but also correlate with vasoconstriction and vasodilation respectively, directly modulating blood vessel architecture and therefore CBF (19).

To gain insights into BOLD mechanisms and by extension into brain activity, it is of interest to investigate longer paradigms of

\footnotetext{
* Correspondence to: N. Just, Centre d'Imagerie Biomédicale - Animal and Technology Core (CIBM-AIT), Lausanne, Switzerland.

E-mail: nathalie.just@epfl.ch
}

a S. Sonnay, J. M. N. Duarte, R. Gruetter

Laboratory for Functional and Metabolic Imaging, École Polytechnique Fédérale Lausanne, Switzerland

b N. Just

Centre d'Imagerie Biomédicale - Animal and Technology Core (CIBM-AIT), Lausanne, Switzerland

c R. Gruetter

Department of Radiology, University of Geneva, Switzerland

d R. Gruetter

Department of Radiology, University of Lausanne, Switzerland

Abbreviations used: BOLD, blood oxygenation level dependent; $f M R I$, functional MRI; ISI, inter-stimulus interval; CBF, cerebral blood flow; CBV, cerebral blood volume; $\mathrm{CMRO}_{2}$, cerebral metabolic rate of oxygen; FOV, field of view; ANOVA, analysis of variance; S1FL, primary somatosensory forelimb; S1HL, primary somatosensory hindlimb; $\mathrm{M1}$, primary motor cortex; $\mathrm{M}$, secondary motor cortex; S2, secondary somatosensory cortex; S1BF, primary somatosensory cortex of the barrel cortex region; NMDA, N-methyl-D-aspartate; SNR, signalto-noise ratio; GE-EPI, gradient echo echo planar imaging; SD, standard deviation. 
activation. Cerebral processing might occur after stimulation (20) and not necessarily following periodic events, as usually provided by BOLD fMRI paradigms. We propose here to introduce prolonged stimulation paradigms over $2 \mathrm{~h}$ for reproducible and localized cortical activation in rats under physiological conditions, as measured by BOLD fMRI at high-field magnetic resonance $(9.4 \mathrm{~T}$ ) and using electrical stimulation of the forepaw.

\section{MATERIALS AND METHODS}

\section{Animals}

All experiments were performed in accordance with the Swiss federal law on animal experimentation and approved by the local authority (EXPANIM-SCAV). Male adult Sprague-Dawley rats ( $n=14,328 \pm 16$ g, from Charles River Laboratoires, L'arbresle, France) were initially anaesthetized with $2 \%$ isoflurane vaporized in $30 \%$ oxygen in air, intubated and ventilated with a pressuredriven ventilator (MRI-1, CWE, Ardmore, PA, USA). A femoral artery and a femoral vein were catheterized for blood sampling and infusions, respectively. The rat was positioned in a homebuilt holder and the rat head was fixed using ear and bite bars. Heart rate, arterial blood pressure and breathing rate were continuously monitored with an animal monitoring system (SA Instruments, Stony Brook, NY, USA). Blood gases were measured using a blood gas analyzer (AVL Compact 3, Diamond Diagnostics, Holliston, MA, USA) and were maintained at physiological levels $\left(P_{\mathrm{a}} \mathrm{CO}_{2}=32-40 \mathrm{~mm} \mathrm{Hg}, \mathrm{pH}=7.32-7.42\right)$. Blood samples were not collected during the stimulation periods. Body temperature was maintained at $37.5 \pm 0.5^{\circ} \mathrm{C}$ with a warm water circulation system based on the feedback obtained from a homebuilt rectal temperature probe. After surgery, anesthesia was switched to $\alpha$-chloralose (Acros Organics, Geel, Belgium) in saline solution ( $80 \mathrm{mg} / \mathrm{kg}$ bolus followed by a continuous intravenous infusion rate of $27 \mathrm{mg} / \mathrm{kg} / \mathrm{h}$ ). Pancuronium bromide ( $2 \mathrm{mg} / \mathrm{kg}$ i.v., Sigma-Aldrich, Basel, Switzerland) was administered 5-10 min before stimulation.

\section{Electrical stimulation}

Two stainless steel electrodes were inserted between the second and third digits of the left forepaw. Electrical stimulation was performed by delivering square pulses ( $0.5 \mathrm{~ms}$ width) using an external stimulator (WPI, Stevenage, UK). A few experimental sessions were dedicated to investigate the responses in terms of activated volume and BOLD amplitude in the contralateral cortex to find the parameters generating the best contrast to be used for long stimulation experiments. Current intensities ranging from 0.5 to $3 \mathrm{~mA}$ at $2 \mathrm{~Hz}$ were gradually applied with resting periods of 8-10 $\mathrm{min}$ in between. For each current level the paradigm was [30 s ON-30 s OFF] over $4 \mathrm{~min}$.

Several forepaw stimulation paradigms with inter-stimulus interval (ISI) 60,30 and $10 \mathrm{~s}$ at constant $(2 \mathrm{~Hz}$ ) or variable stimulus frequency (2-3 Hz switched every $5 \mathrm{~min}$ ) and constant current ( 2.5 or $3 \mathrm{~mA}$ ) were applied and repeated for $30 \mathrm{~min}$, $1 \mathrm{~h}, 1.5 \mathrm{~h}$ or $2 \mathrm{~h}$ for optimization of the prolonged BOLD response. Stimulus frequencies of 2 and $3 \mathrm{~Hz}$ were chosen based on previous studies $(4,8,21)$ showing maximal BOLD and CBF responses. Each experiment consisted of 5 min baseline (prestimulus period $0-5 \mathrm{~min}$ ), followed by the stimulation period and 5 min recovery period (post-stimulus period). ISI remained unchanged within each experiment.

\section{fMRI experiments}

fMRI experiments were performed on an actively shielded 9.4 $\mathrm{T} / 31 \mathrm{~cm}$ horizontal bore magnet (Magnex Scientific, Abingdon, UK), interfaced to a DirectDrive console (Agilent Technologies, Palo Alto, CA, USA) and equipped with $12 \mathrm{~cm}$ gradients (400 $\mathrm{mT} / \mathrm{m}$ in $120 \mu \mathrm{s})$, using a homebuilt proton quadrature transmit/receive surface coil resonating at $400 \mathrm{MHz}$. The rat brain was placed at the isocenter and $T_{2}$-weighted fast spin echo multi slice images (TR/echo spacing (ESP)/echo train length $(E T L)=4$ $\mathrm{s} / 10 \mathrm{~s} / 8$; field of view $(\mathrm{FOV})=30 \times 30 \mathrm{~mm}^{2}$; matrix $=128 \times 128$; slice thickness $=1 \mathrm{~mm}$ ) were acquired for anatomical reference. Shimming was performed with FAST(EST)MAP (22), resulting in water half linewidths of $16-21 \mathrm{~Hz}$ in a $270 \mu \mathrm{L}$ volume. The BOLD response was measured with single shot gradient echo echo planar imaging (GE-EPI) (TR/TE $=2.5 \mathrm{~s} / 25 \mathrm{~ms} ; \mathrm{FOV}=30 \times 30 \mathrm{~mm}^{2}$; matrix $=64 \times 64$; slice thickness $=1 \mathrm{~mm}$; six slices; bandwidth $=$ $300-350 \mathrm{kHz}$ ). Echo realignment was performed using a built-in reference scan (23).

\section{Data analysis}

Images were first reconstructed using homebuilt MATLAB routines (MathWorks, Natick, MA, USA) and motion correction was performed using SPM8 (Statistical Parametric Mapping, London, UK). No other correction or filtering methods (i.e. baseline and drift (24)) were applied. Activation $t$-value maps were computed on a voxel-wise basis from the comparison between the experimental fMRI data and the applied paradigm schemes for either the first or last $7 \mathrm{~min}$ of paradigm (relatively to the pre-stimulus period or the post-stimulus period, respectively) using STIMULATE (25). Only clusters including at least three voxels were considered significant. While the mapping threshold was set at $p<$ 0.01 for current-dependent signal experiments, it was at $p \leq 0.3$ for activated volume quantification in long stimulation data, because of the overall decrease of $t$-values at the end of the stimulation (for mapping purposes, this decrease of $t$-values required thresholding at a lower $p$-value). Activated brain areas were identified by comparison with the rat brain atlas (26). Timecourses from the activated regions in the contralateral cortex were extracted and used to calculate the relative BOLD response, $t$-values and volume of activation. The relative BOLD response was computed relatively to the pre-stimulus period as follows:

$$
\text { BOLD }_{\text {relative, } i}=\frac{\text { stimulation }_{i}-\overline{\text { baseline }_{0-5 \text { min }}}}{\overline{\text { baseline }}_{0-5 \text { min }}}
$$

where $i$ is the scan number (TR $=2.5 \mathrm{~s}$ ).

Repeated measure analysis of variance (ANOVA) was performed to assess significance between means of BOLD response amplitude, $t$-values and volumes of activation ( $p<0.05$ was considered as significant). Post hoc analysis after ANOVA was performed using Bonferroni's multiple comparison test. Data are presented as mean \pm standard deviation (SD).

\section{RESULTS}

Effect of current intensity on the BOLD amplitude and activated volume

As a starting point, we verified that our experimental conditions were in agreement with previous literature findings using short paradigms, for which different current values were tested 
at $2 \mathrm{~Hz}$. Strong and robust activation was detected in four to six consecutive slices in the contralateral primary somatosensory forelimb (S1FL) and primary motor cortex (M1) with current intensity above $1 \mathrm{~mA}$ in all rats $(n=6)$ (Fig. $1(\mathrm{~A})$ ). Figure $1(\mathrm{~B})$ shows a typical BOLD response extracted at $2.5 \mathrm{~mA}$. A gradual increase in current intensities corresponded to an increase in BOLD amplitudes with a plateau starting at $2 \mathrm{~mA}$ and ranging from $5.9 \pm 2.0 \%$ to $6.7 \pm 1.4 \%$ ( $p<0.05$ compared with $1 \mathrm{~mA}$, Fig. 1(C)). The activated volume increased with current intensity, and larger activated volumes ranging from $22 \pm 7$ to $30 \pm 13 \mathrm{~mm}^{3}$ were observed from 2 to $3 \mathrm{~mA}$ ( $p<0.01$ compared with $1 \mathrm{~mA}$, Fig. 1 (D)). $t$-values extracted from the activated regions also increased with the applied current intensity, reaching a plateau at $2 \mathrm{~mA}$ with values ranging from $6.8 \pm 0.8$ to $7.1 \pm 1.4$ ( $p<0.01$ compared with $1 \mathrm{~mA}$, Fig. 1(E)). According to these observations, current values of 2.5 or $3 \mathrm{~mA}$ were therefore chosen for long stimulation experiments.

\section{Prolonged BOLD signal with ISI over $\mathbf{1}$ and $\mathbf{2} \mathbf{h}$}

Continuous stimulation of the left forepaw at $3 \mathrm{~Hz}$ and $3 \mathrm{~mA}$ resulted in the well-established loss of the BOLD signal after approximately $15 \mathrm{~min}$ ( $n=4$, Fig. 2(A)). Therefore, to determine the effect of using an ISI, we continuously stimulated the left forepaw and added ISI amounting to 50 or $25 \%$ of the total time. Several paradigms at constant current and frequency ( $3 \mathrm{~mA}, 3$ $\mathrm{Hz}$ ) with different ISIs were individually and reproducibly applied for $1 \mathrm{~h}$ in the left forepaw, from the longest to the shortest ISI: [60 s ON-60 s OFF] (Fig. 2(B)); [30 s ON-30 s OFF] (Fig. 2(C)); [30 s ON-10 s OFF] (Fig. 2(D)). This last paradigm [30 s ON-10 s OFF] was then repeated for $1 \mathrm{~h}$ by switching the frequency of stimulation between 2 and $3 \mathrm{~Hz}$ every 5 min (Fig. 2(E)). Although all resulted in $1 \mathrm{~h}$ prolonged positive BOLD responses mimicking the applied paradigms and reflecting cerebral activation in the right hemisphere, the amplitudes of the BOLD responses over time tended to remain higher in Figure 2(E) ( 5\%) than in Figure 2(D) ( 3\%).

To determine the effect of variable stimulus frequency, the [30 s ON-10 s OFF] paradigm was performed over $2 \mathrm{~h}$ at $2.5 \mathrm{~mA}$ in the left forepaw and the stimulus frequency was varied every 5 min between 2 and $3 \mathrm{~Hz}(n=5)$ (Fig. 3) or maintained constant $(2 \mathrm{~Hz})(n=5)$. The average BOLD amplitude reached a maximum of $3.6 \pm 1.0 \%$ and $2.8 \pm 0.7 \%$ after the onset of the stimulation for variable and constant frequency conditions, respectively (Fig. 3(B)). After $15 \mathrm{~min}$, the average BOLD amplitude dropped to a steady state of $1.3 \pm 0.2 \%$ and $0.8 \pm 0.3 \%$ for variable and constant frequency conditions, respectively, lasting until the end of the stimulation and reflecting persistent neuronal activation (Fig. 3(B)). Therefore, although both conditions resulted in localized and prolonged positive BOLD responses over the entire stimulation period, the steady-state amplitude of the signal significantly increased on average when the stimulus frequency was varied ( $t$-test, $p<0.05$ ). A drift in the BOLD time course was moreover observed, because no correction to the baseline was applied (Fig. 3(C)).

Although not different, the activated volume $\left(\mathrm{mm}^{3}\right)$ in the contralateral cortex at the end of the stimulation in the constant frequency condition was associated with larger SDs compared with variable frequency, demonstrating increased variability during stimulation across rats. More precisely, the fraction of remaining activated volume was $1.0 \pm 0.3$ at variable frequency and $1.0 \pm 0.8$ at constant frequency. Moreover, a significant decrease ( $t$-test, $p<0.05)$ in $t$-values of $62 \pm 19 \%$ and $57 \pm 13 \%$ was observed after the end of the stimulation compared with the beginning for variable and constant frequency conditions, respectively.
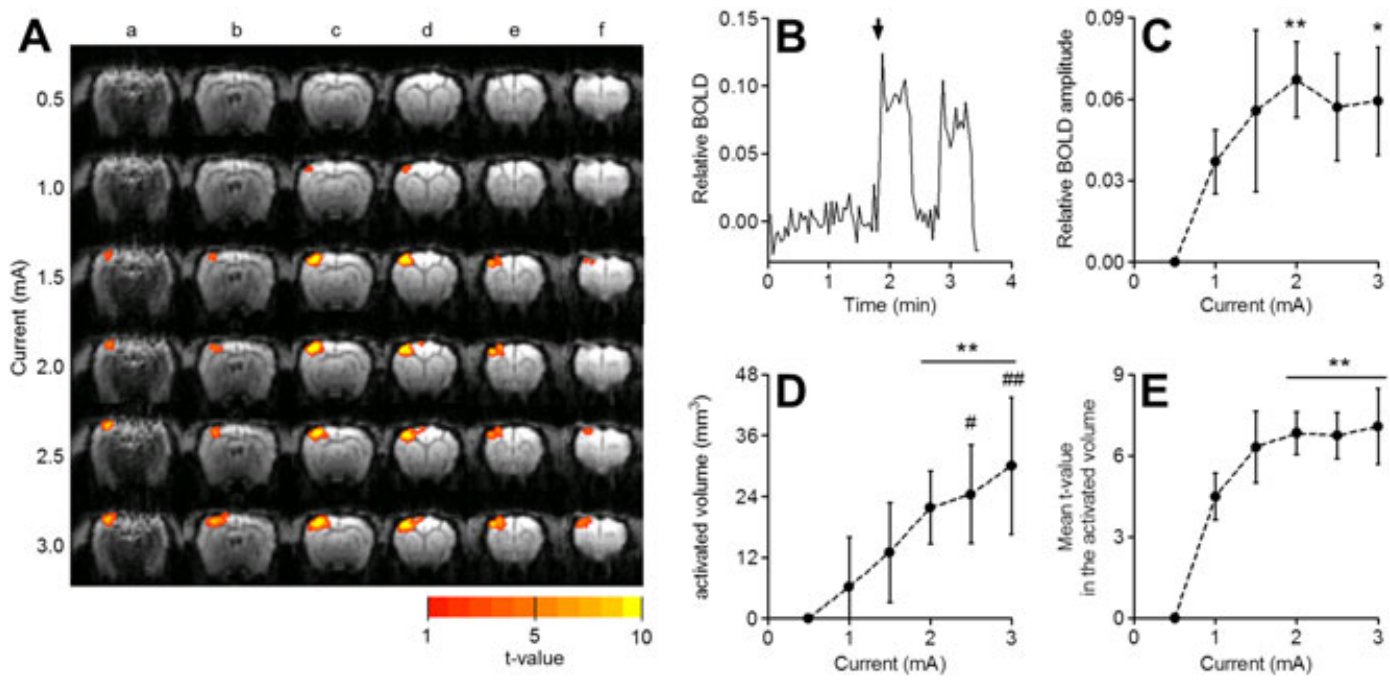

Figure 1. Effect of current on BOLD amplitude and activated volume during forepaw stimulation. (A) Typical activation $t$-value maps in one representative rat (1 mm thickness) showing significant activation of contralateral S1, M1 and M2 overlaid on GE-EPI images in six slices (a, bregma -2.8; b, bregma -1.8 ; $c$, bregma -0.8 ; $d$, bregma +0.2 ; e, bregma $+1.2 ;$, bregma +2.2 ) following electrical stimulation ( $30 \mathrm{~s}$ ON-30 s OFF repeated for 4 $\mathrm{min}$ at $2 \mathrm{~Hz}$ ) of the left forepaw at different current intensities from 0.5 to $3 \mathrm{~mA}$. Only clusters including at least three pixels were considered significant $(p<0.01)$. (B) Typical relative BOLD signal extracted from slices $d$ in (A) at $2.5 \mathrm{~mA}$. The black arrow indicates the beginning of the stimulation. (C) Current-dependent relative BOLD response amplitude in activated volume computed as follows: BOLD $=\overline{\text { stimulation }_{k}}-\overline{\overline{I S I}_{k}}$ where $k$ is each block of the paradigm [30 s ON-30 s OFF]. In other words, the calculation of the relative BOLD amplitude was based on the difference between the mean of the signal corresponding to $30 \mathrm{~s}$ ON and the mean of the following ISI (30 s OFF). (D) Current-dependent activated volume (mm ${ }^{3}$ ). (E) Average $t$-values extracted from the activated regions at different current intensities. Data are mean $\pm \mathrm{SD}$ of $n=6$. ${ }^{*} p<0.05,{ }^{* *} p<0.01$ compared with $1 \mathrm{~mA}$; $p<0.05$, ${ }^{\# \#}$ $p<0.01$ compared with $1.5 \mathrm{~mA}$ with repeated measurements ANOVA. 

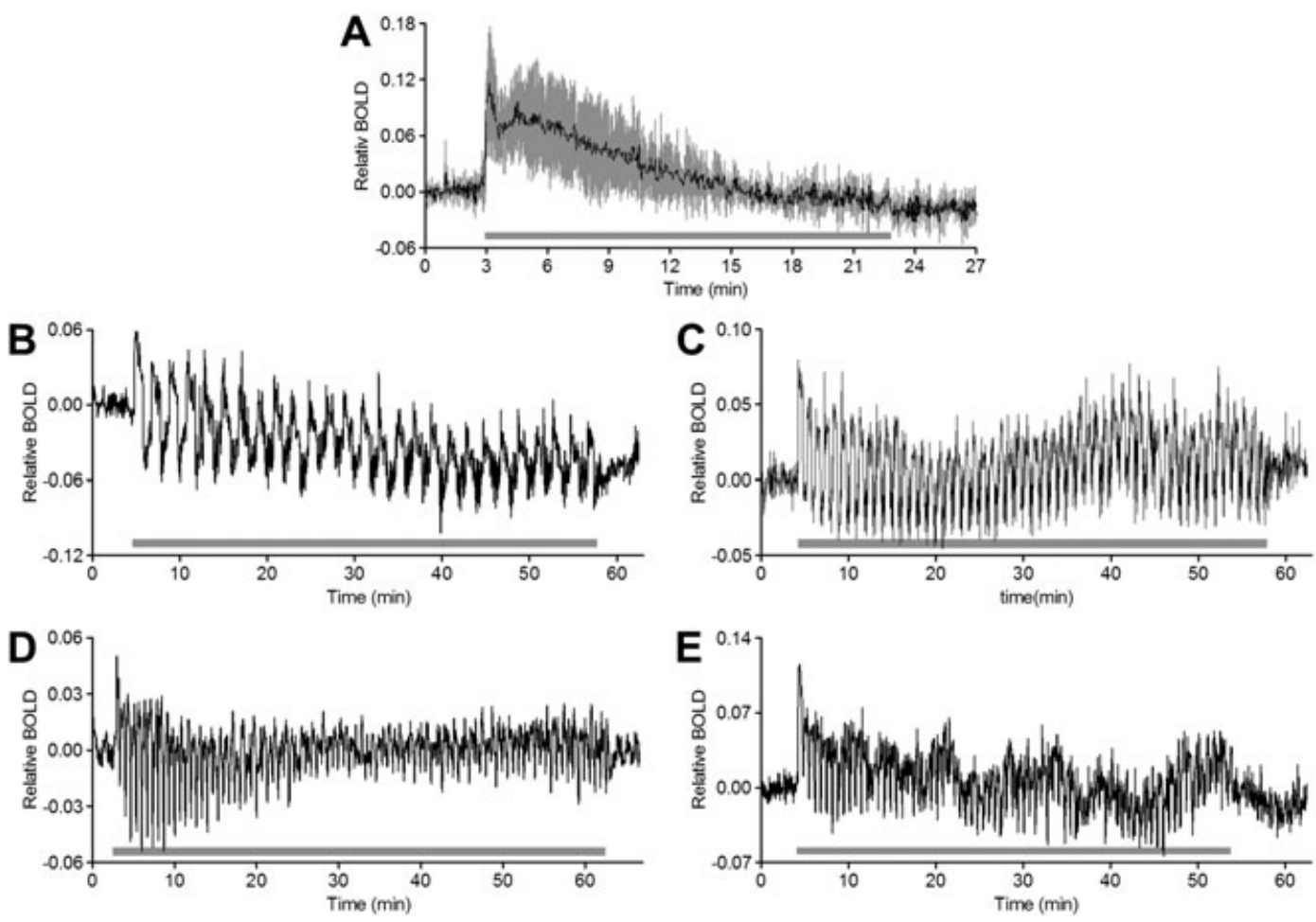

Figure 2. BOLD fMRI responses at different ISIs. (A) Relative average BOLD response $(n=4)$ during 20 min of continuous stimulation at $3 \mathrm{~Hz}$ and $3 \mathrm{~mA}$. (B)-(E) Relative BOLD response in the contralateral cortex acquired during $1 \mathrm{~h}$ stimulation at $3 \mathrm{~mA}$ with the following paradigms: (B) $60 \mathrm{~s}$ ON-60 s OFF at $3 \mathrm{~Hz}$; (C) 30 s ON-30 s OFF at $3 \mathrm{~Hz}$; (D) 30 s ON-10 s OFF at $3 \mathrm{~Hz}$; (E) 30 s ON-10 s OFF at 2-3 Hz switched every 5 min. The shaded area represents the entire stimulation period.

\section{Other activated brain areas}

Contralateral S1FL remained activated during the whole stimulation period $(2 \mathrm{~h})$ in all rats $(n=10)$, whereas BOLD responses in $M 1$ were detected until the end in nine out of ten rats. Secondary motor cortex (M2) and secondary somatosensory cortex (S2) were activated to a lesser extent than $\mathrm{M} 1$ and not in all rats. BOLD responses in primary somatosensory cortex of the hindlimb region $(\mathrm{S} 1 \mathrm{HL})$ were observed in six rats (Table 1). Ipsilateral cortical activation was also observed in six rats, but not during the entire stimulation period, and smaller in amplitude, as compared with the contralateral side (Fig. 4(A), (B)). No significant BOLD signal was detected in deeper brain areas, such as the striatum (Fig. 4(B)).

\section{Absence of physiology deterioration during long electrical stimulation}

Arterial blood pressure and heart rate were unaltered during the whole experiment (Fig. 5(A), (B)). Blood samples were collected before and after the stimulation period and indicated that $P_{\mathrm{a}} \mathrm{CO}_{2}$ and $\mathrm{pH}$ remained in the physiological range after stimulation (Fig. $5(C)$ ), suggesting that the prolonged stimulation protocol does not alter blood homeostasis.

The absolute average motion over the entire stimulation time ( $2 \mathrm{~h}$ ) was $0.04 \pm 0.07,0.48 \pm 0.34$ and $0.07 \pm 0.09 \mathrm{~mm}$ in the $x, y$ and $z$ directions, respectively $(n=10)$. The water linewidth at half height in the volume of interest was $10 \pm 13 \%(n=10)$ larger at the end of the fMRI protocol compared with the beginning of the experiment. The signal-to-noise ratio (SNR) of the GE-EPI images decreased in absolute value by $4.5 \pm 2.7 \%(n=10)$ after the end of acquisition.

\section{DISCUSSION}

The present study confirmed the rapid hemodynamic adaptation to continuous sensorial stimulation as determined using BOLD $\mathrm{fMRI}$. It also demonstrated that using a small ISI resulted in a prolonged BOLD activation over $2 \mathrm{~h}$ in the forepaw-associated cortex, suggesting reduced hemodynamic adaptation effects compared with continuous stimulation. In addition, the present results indicate that modulation of the applied stimulus frequency not only decreased activated volume variability, but was also associated with higher steady-state BOLD amplitude compared with prolonged stimuli at constant frequency. The well-resolved individual BOLD responses over the entire stimulation period demonstrate the robustness of the protocol. Therefore, the long stimulation paradigm presented here might allow more extensive studies providing insights into unknown features of the neurovascular coupling, brain activity and complex nature of the BOLD signal.

We performed electrical stimulations using short paradigms at 2.5-3 $\mathrm{mA}$ that resulted in robust cortical activation with maximum BOLD responses and activated volume in line with previous reports $(4,7-9,21)$. Then, continuous stimulation of the left forepaw at $3 \mathrm{~Hz}$, performed under physiological conditions, resulted in a rapid decay of the underlying BOLD signal after about 10-15 min. This adaptation to the delivered stimulus possibly reflects a mismatch between $\mathrm{CMRO}_{2}$ and $\mathrm{CBF}$ (2) and reduced neuronal firing $(1,3,6)$.

To our knowledge, this study is the first report of a localized and prolonged BOLD signal that persisted for $2 \mathrm{~h}$, in contrast to continuous stimulation (6). This was achieved upon electrical stimulation of the forepaw with an ISI, allowing for the 

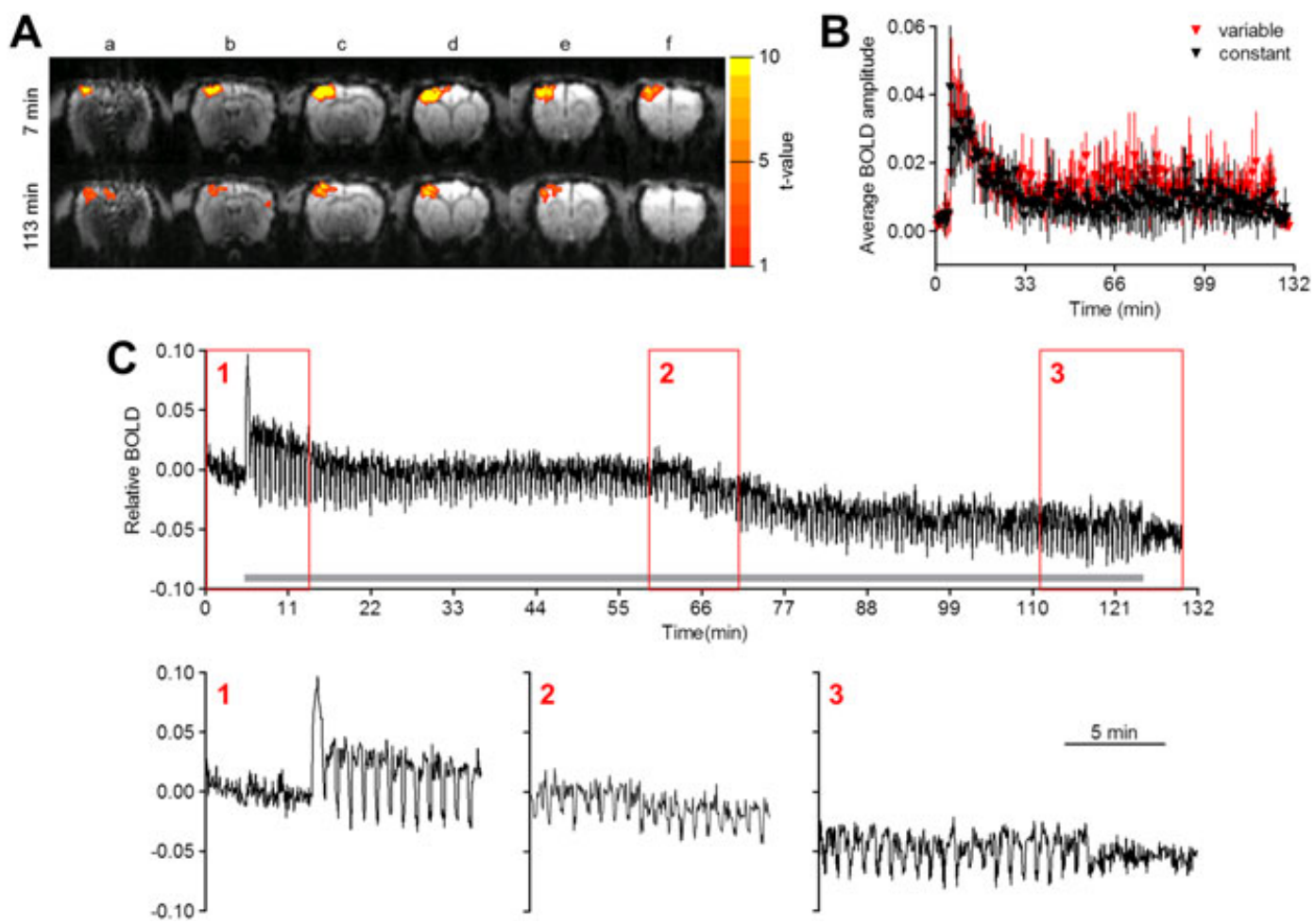

Figure 3. Electrical stimulation of the left forepaw for $2 \mathrm{~h}$ with variable frequency. (A) Representative activation $t$-value map in one rat showing activation of contralateral cortex overlaid on GE-EPI images ( $1 \mathrm{~mm}$ thickness) in six slices (a, bregma $-2.8 ; \mathrm{b}$, bregma $-1.8 ; \mathrm{c}, \mathrm{bregma}-0.8$; $\mathrm{d}$, bregma +0.2 ; e, bregma +1.2; f, bregma +2.2) after 7 and $113 \mathrm{~min}$ of left forepaw electrical stimulation at variable frequency ( $30 \mathrm{~s}$ ON-10 s OFF repeated for $2 \mathrm{~h}$ at 2.5 $\mathrm{mA}$ and $2-3 \mathrm{~Hz}$ switched every $5 \mathrm{~min}$ ). Only clusters including at least three pixels were considered as significant. (B) Average of relative BOLD amplitudes across rats during $2 \mathrm{~h}$ stimulation for both constant and variable frequency conditions: BOLD $\bar{k}=\overline{\text { stimulation }_{k}}-\overline{\operatorname{ISI}_{k}}$ where $k$ is each block of the paradigm [30 s ON-10 s OFF]. In other words, the calculation of the relative BOLD amplitude was based on the difference between the mean of the signal corresponding to $30 \mathrm{~s}$ ON and the mean of the following ISI (10 s OFF). Data are shown as average over $40 \mathrm{~s}$ (mean \pm SD). (C) Relative BOLD signal observed during $2 \mathrm{~h}$ stimulation, as in (A). The shaded area represents the stimulation period. Regions 1-3 in red rectangles are expanded below.

Table 1. Contralateral activated areas observed at the beginning (up to $7 \mathrm{~min}$ ) and at the end (from $113 \mathrm{~min}$ ) onwards of $2 \mathrm{~h}$ stimulation, and average SNR of the GE-EPI images between these two periods of time

\begin{tabular}{|cclcc} 
Stimulus frequency & Rat & \multicolumn{1}{c}{7 min } & \multicolumn{1}{c}{113 min } & Average SNR \\
\hline \multirow{3}{*}{ Variable $(2-3 \mathrm{~Hz})$} & 1 & $\mathrm{~S} 1 \mathrm{FL}, \mathrm{M} 1$ & $\mathrm{~S} 1 \mathrm{FL}, \mathrm{M} 1, \mathrm{~S} 2$ & $17.5 \pm 0.7$ \\
& 2 & $\mathrm{~S} 1 \mathrm{FL}, \mathrm{M} 1$ & $\mathrm{~S} 1 \mathrm{FL}, \mathrm{M} 1, \mathrm{M} 2, \mathrm{~S} 2$ & $22.2 \pm 0.5$ \\
& 3 & $\mathrm{~S} 1 \mathrm{FL}, \mathrm{M} 1$ & $\mathrm{~S} 1 \mathrm{FL}, \mathrm{M} 1$ & $13.7 \pm 0.1$ \\
& 4 & $\mathrm{~S} 1 \mathrm{FL}, \mathrm{M} 1, \mathrm{M} 2, \mathrm{~S} 2, \mathrm{~S} 1 \mathrm{HL}$ & $\mathrm{S} 1 \mathrm{FL}, \mathrm{M} 1, \mathrm{~S} 2$ & $15.3 \pm 0.6$ \\
& 5 & $\mathrm{~S} 1 \mathrm{FL}, \mathrm{M} 1, \mathrm{M} 2$ & $\mathrm{~S} 1 \mathrm{FL}, \mathrm{M} 1, \mathrm{M} 2, \mathrm{~S} 2, \mathrm{~S} 1 \mathrm{HL}$ & $18.1 \pm 1.1$ \\
& & & $17.4 \pm 3.0$ \\
Constant $(2 \mathrm{~Hz})$ & 6 & $\mathrm{~S} 1 \mathrm{FL}, \mathrm{M} 1$ & $\mathrm{~S} 1 \mathrm{FL}, \mathrm{M} 1$ & $17.5 \pm 0.5$ \\
& 7 & $\mathrm{~S} 1 \mathrm{FL}, \mathrm{M} 1, \mathrm{M} 2, \mathrm{~S} 2, \mathrm{~S} 1 \mathrm{HL}$ & $\mathrm{S} 1 \mathrm{FL}, \mathrm{M} 1, \mathrm{M} 2$ & $8.3 \pm 0.0$ \\
& 8 & $\mathrm{~S} 1 \mathrm{FL}, \mathrm{M} 1, \mathrm{~S} 1 \mathrm{HL}$ & $\mathrm{S} 1 \mathrm{FL}, \mathrm{M} 1, \mathrm{M} 2, \mathrm{~S} 2, \mathrm{~S} 1 \mathrm{HL}$ & $15.0 \pm 0.7$ \\
& 9 & $\mathrm{~S} 1 \mathrm{FL}$ & $\mathrm{S} 1 \mathrm{FL}, \mathrm{M} 1, \mathrm{M} 2, \mathrm{~S} 2, \mathrm{~S} 1 \mathrm{HL}$ & $16.7 \pm 0.9$ \\
& 10 & $\mathrm{~S} 1 \mathrm{FL}, \mathrm{M} 1, \mathrm{M} 2, \mathrm{~S} 2, \mathrm{~S} 1 \mathrm{HL}$ & $\mathrm{S} 1 \mathrm{FL}, \mathrm{M} 1, \mathrm{M} 2$ & $13.3 \pm 0.3$ \\
& & & $14.1 \pm 2.5$ \\
\hline
\end{tabular}

refractoriness of the BOLD signal (27). Different ISIs $(60,30$ and $10 \mathrm{~s})$ were tested and all allowed to persistently elicit a BOLD response until the end of the stimulation, which lasted for $2 \mathrm{~h}$. The typical BOLD response was characterized by a decay in the relative BOLD signal to a plateau, which lasted for the rest of the stimulation period. This plateau was reached after 15-20 min, which corresponded to the time at which the BOLD response was lost in continuous stimulation paradigms (see Figs. 2(A) and $3(B))$. Interestingly, in contrast to continuous stimulation (6), trigeminal nerve stimulation with ISI was also able to elicit BOLD responses that persisted for $2 \mathrm{~h}$ (not shown), suggesting that similar paradigms can be used in other brain areas.

Thalamic adaptation is associated with a decrease in action potentials during sustained stimulation, reducing therefore the transmission efficiency of the stimulus to the cortex (28). Therefore, to minimize adaptation of the sensory system to a constant 


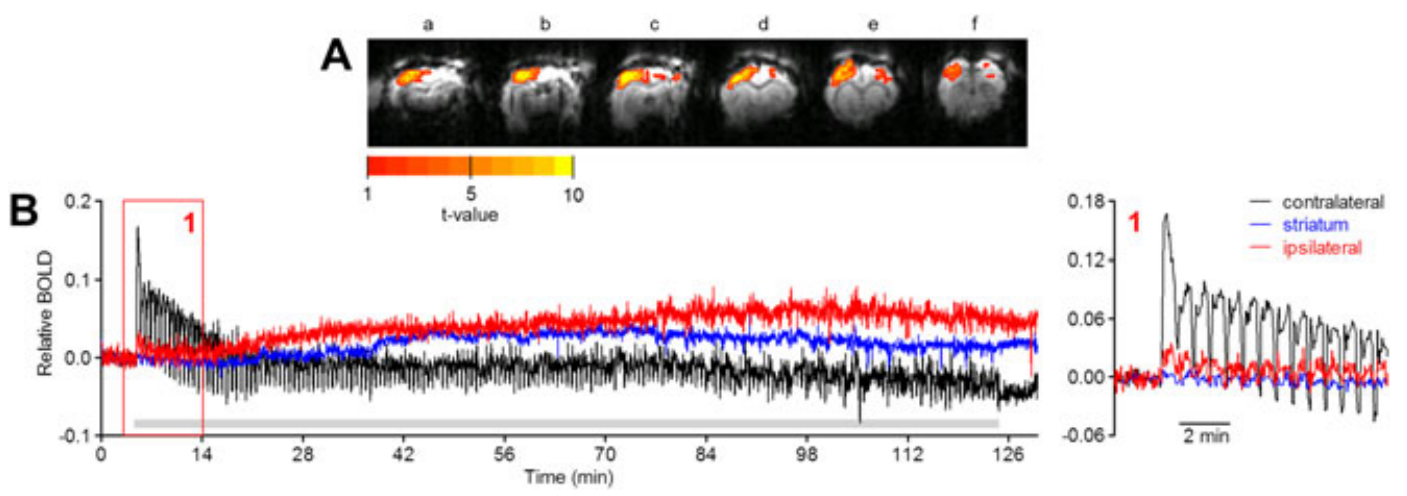

Figure 4. Ipsilateral activation following electrical stimulation of the left forepaw for $2 \mathrm{~h}$. (A) Representative activation $t$-value map in one representative rat showing initial activation of contralateral and ipsilateral cortex overlaid on GE-EPI images ( $1 \mathrm{~mm}$ thickness) in six slices (a, bregma -2.8 ; $b$, bregma -1.8 ; c, bregma -0.8 ; d, bregma +0.2 ; e, bregma +1.2; f, bregma +2.2) after 7 min of left forepaw electrical stimulation at variable frequency (30 s ON-10 s OFF repeated for $2 \mathrm{~h}$ at $2.5 \mathrm{~mA}$ and $2-3 \mathrm{~Hz}$ switched every $5 \mathrm{~min}$ ). Only clusters including at least three pixels were considered as significant. (B) Relative BOLD signals observed during $2 \mathrm{~h}$ stimulation, as in (A), in the contralateral and ipsilateral sides and bilateral striatum. The ipsilateral activation is smaller in amplitude and lasts for $14 \mathrm{~min}$, while the contralateral response persists during the entire stimulation period. No significant BOLD response was detected in the striatum. The expansion on the left shows the expansion of the first 14 min. The shaded area represents the stimulation period.
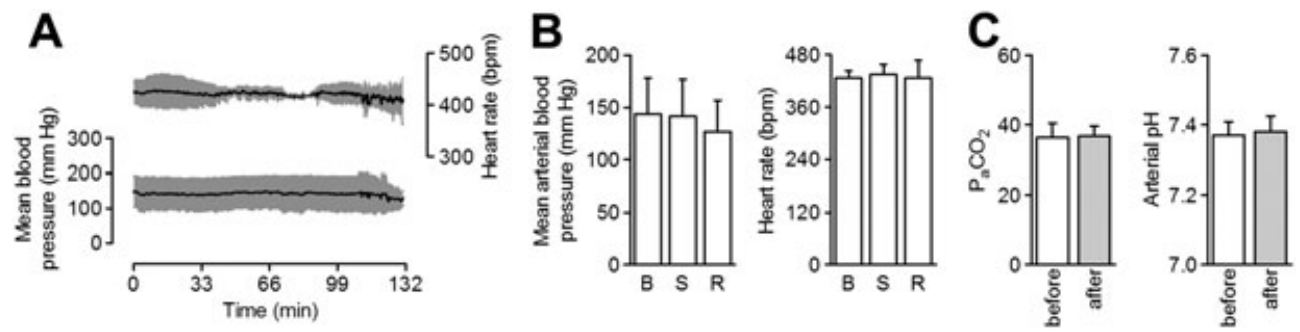

Figure 5. Physiology during $2 \mathrm{~h}$ stimulation of the left forepaw. (A) Heart rate and mean arterial blood pressure during the entire stimulation period. Sampling rate was $1 \mathrm{~s}$ and data are shown as average over $30 \mathrm{~s}(n=3)$. (B) Average of heart rate and blood pressure during baseline (B), stimulation (S) and recovery $(\mathrm{R})$ periods. $(\mathrm{C})$ Arterial $\mathrm{CO}_{2}$ pressure $(n=10)$ and $\mathrm{pH}(n=10)$ before and after the stimulation period. Data are mean \pm SD.

stimulus input $(2,29)$, the frequency of stimulation was modulated every 5 min between 2 and $3 \mathrm{~Hz}$. These frequencies give the maximum response in terms of activation area (7), BOLD $(4,8,21)$ and CBF responses $(8,21)$ under $\alpha$-chloralose anesthesia. However, no statistical differences in the fraction of remaining activated volume in the contralateral cortex at the end of the stimulation and in activated anatomical regions were detected between variable and constant frequency conditions. Nevertheless, the variance observed in the remaining activated volume was larger in the constant frequency condition, suggesting reduced inter-individual variability in thalamo-cortical projecting fibers of the stimulated pathway $(30,31)$ in the variable frequency condition. Moreover, higher steady-state BOLD amplitude was observed when varying the stimulus frequency, reflecting possible response recovery from different stimulus inputs (2).

A rapid decline in BOLD amplitude was followed by a steadystate plateau lasting until the end of the stimulation. The rapid BOLD increase at the onset of the stimulation or overshoot has been observed both in the primary somatosensory cortex of the barrel cortex region (S1BF) (6) and in humans (3), and the plateau in S1BF (6). The first adaptative mechanism might reflect rapid and large neuronal hyperpolarization (13), modulation of the responses produced by thalamo-cortical fibers that drive action potentials $(30,31)$ or uncoupling between $\mathrm{CBF}$ and $\mathrm{CMRO}_{2}$ $(2,14)$. The second might rather be associated with neuronal fatigue $(3,6)$, as the addition of ISI allows the BOLD signal to recover from the hyperemic mismatch (27), without excluding any hemodynamic coupling alterations. Fluctuations observed in the BOLD steady-state plateau result from averaging BOLD responses over a large activated area, where different local $\mathrm{CMRO}_{2}$ values, $\mathrm{CBFs}$ and cerebral blood volumes (CBVs) might occur across averaged voxels.

Although the responses were mainly localized in the contralateral S1FL, activation of contralateral M1, M2 and S2, as well as other smaller cortical structures, was often observed during prolonged stimulation. However, these areas were not consistently detected. Motor cortex activation might result from small vibrations of the paw upon stimulation of the nonnociceptive antidromic stimulation of the motor nerve fibers (32). S1HL activation is likely the result of the thalamo-cortical afferent fibers, involved in stimulation information processing, connecting the ventral posterolateral thalamic nucleus and S1FL and probably encompassing other somatosensory cortices, such as S1HL (30). Similarly, ipsilateral activation might reflect the inter-hemispherically connecting function of the corpus callosum (30). Since fMRI indirectly assesses neuronal activity, by taking advantage of the uncoupling between CBF, $\mathrm{CBV}$ and $\mathrm{CMRO}_{2}$ (33), the hemodynamic response might be extended to neighboring brain regions to provide enough energy supply for the maintenance of the overall brain homeostasis (34). Despite the presence of cortico-striatal connections (35), $T_{2}{ }^{*}$-weighted signal in the striatum did not result 
in a significant BOLD response following the applied paradigm. One reason could be the low sensitivity in this particular region. Indeed, a surface coil is more efficient for brain areas close to the surface and is therefore optimized for studies in cortex. In addition, the BOLD response is about $5-10 \%$ of the baseline signal. Therefore, if the noise level in the other brain regions far from the cortex is much larger than the BOLD response, the signal becomes hard to detect. Moreover in a recent article, Shulman et al. highlight the fact that changes in BOLD signal induced by sensory stimulation might be many times smaller than the high and ubiquitous resting level of neuronal activity induced by light anesthesia (i.e. $\alpha$-chloralose). Therefore, the absence of BOLD response underlying sensory stimulation in a given voxel cannot be directly interpreted as evidence for the neurons not supporting the task (16).

The average $t$-values extracted from the activated region decreased significantly after $2 \mathrm{~h}$ stimulation, reflecting not only the steady-state plateau, but also shim degradation ( 10\%) and decreased SNR, despite the good quality of the acquired data. Changes in animal physiology, namely blood pressure, heart rate, arterial $\mathrm{pH}$ and $\mathrm{P}_{\mathrm{a}} \mathrm{CO}_{2}$, that directly control the cerebrovascular system were unlikely to contribute to the reduction in $t$-values, since they remained unchanged throughout the stimulation period. In addition, physiological data suggest that the stimulation applied in the present study was innocuous, not inducing changes in the vascular system, particularly blood pressure and heart rate $(6,8,36)$.

All together, these observations suggest that adding an ISI as small as $10 \mathrm{~s}$, as well as modulating the frequency at regular intervals, results in a prolonged and localized BOLD response, reflecting neuronal activity with less variability in the fraction of remaining activated volume and higher BOLD amplitude. Investigating brain functionality requires a refined control of animal physiology (19), nociception (36), and neuronal (6) and hemodynamic $(2,14)$ adaptations to the stimulus, which was achieved in the present study. Continuous stimulation paradigms at $1 \mathrm{~Hz}$ have already been performed in S1BF (6), but during a short period of time (10-15 min), after which the BOLD signal tended to decrease, probably due to both neuronal and hemodynamic adaptations, and to be less reproducible across animals. Moreover, continuous stimulation might lead to a concomitant increase in blood pressure and heart rate, representative of nociceptive mechanisms (36). Therefore, optimal physiological conditions are necessary for not only BOLD signal detection $(19,33)$, but also biologically meaningful measures. The long stimulation paradigm presented here mimics rhythmic environmental patterns, with different sensorial stimulation inputs, as often encountered in natural settings, such as walking or whisking (20). An animal model or a human is unlikely to be continuously exposed to the same stimulus for a long period of time, but will rather be confronted with different alternatives. For these reasons, the proposed protocol is likely a representative model of what the brain experiences when exposed to lasting external stimuli and is suitable for long experimental protocols.

\section{CONCLUSION}

We conclude that prolonged and localized BOLD response can reproducibly be observed over $2 \mathrm{~h}$ by using an ISI of $10 \mathrm{~s}$, achieving a negligible reduction of the size of the activated volume and less inter-subject variability in the remaining activated volume, and reaching higher steady-state BOLD amplitude when frequency of stimulation is varied. The robustness of such a BOLD fMRI paradigm is likely to enable a new whole brain functional analysis, beyond contralateral S1, S2, M1 and M2 maps.

\section{Conflict of Interest}

There is no conflict of interest to declare.

\section{Acknowledgements}

This work was supported by Centre d'Imagerie Biomédicale (CIBM) of the UNIL, UNIGE, HUG, CHUV, EPFL, the Leenaards and Jeantet Foundations and Swiss National Science Foundation (SNF) grant No 149983. S. Sonnay was supported by the National Competence Center in Biomedical Imaging (NCCBI), and J. M. N. Duarte by SNF grant No 148250. The authors thank Jaquelina Romero, Mario Lepore, Corina Berset and Anne-Catherine Clerc for the technical support.

\section{REFERENCES}

1. Janz C, Heinrich SP, Kornmayer J, Bach M, Hennig J. Coupling of neural activity and BOLD fMRI response: new insights by combination of fMRI and VEP experiments in transition from single events to continuous stimulation. Magn. Reson. Med. 2001; 46(3): 482-486.

2. Moradi F, Buxton RB. Adaptation of cerebral oxygen metabolism and blood flow and modulation of neurovascular coupling with prolonged stimulation in human visual cortex. Neuroimage 2013; 82: 182-189.

3. Uludağ K. Transient and sustained BOLD responses to sustained visual stimulation. Magn. Reson. Imaging 2008; 26(7): 863-869.

4. Huttunen JK, Gröhn O, Penttonen M. Coupling between simultaneously recorded BOLD response and neuronal activity in the rat somatosensory cortex. Neuroimage 2008; 39(2): 775-785.

5. Just N, Petersen C, Gruetter R. BOLD responses to trigeminal nerve stimulation. Magn. Reson. Imaging 2010; 28(8): 1143-1151.

6. Just N, Xin L, Frenkel H, Gruetter R. Characterization of sustained BOLD activation in the rat barrel cortex and neurochemical consequences. Neuroimage 2013; 74: 343-351.

7. Sanganahalli BG, Herman P, Hyder F. Frequency-dependent tactile responses in rat brain by fMRI. NMR Biomed. 2008; 21(4): 410-416.

8. Silva AC, Lee SP, Yang G, ladecola C, Kim SG. Simultaneous blood oxygenation level-dependent and cerebral blood flow functional magnetic resonance imaging during forepaw stimulation in the rat. J. Cereb. Blood Flow Metab. 1999; 19(8): 871-879.

9. Spenger C, Josephson A, Klason T, Hoehn M, Schwindt W, Ingvar M, Olson L. Functional MRI at 4.7 tesla of the rat brain during electric stimulation of forepaw, hindpaw, or tail in single- and multislice experiments. Exp. Neurol. 2000; 166(2): 246-253.

10. Hyder F, Chase JR, Behar KL, Mason GF, Siddeek M, Rothman DL, Shulman RG. Increased tricarboxylic acid cycle flux in rat brain during forepaw stimulation detected with $1 \mathrm{H}[13 \mathrm{C}]$ NMR. Proc. Natl. Acad. Sci. U. S. A. 1996; 93(15): 7612-7617.

11. Mangia S, Tkác I, Gruetter R, Van de Moortele PF, Maraviglia B, Uğurbil K. Sustained neuronal activation raises oxidative metabolism to a new steady-state level: evidence from ${ }^{1} \mathrm{H}$ NMR spectroscopy in the human visual cortex. J. Cereb. Blood Flow Metab. 2007; 27(5): 1055-1063.

12. Schaller B, Xin L, O'Brien K, Magill AW, Gruetter R. Are glutamate and lactate increases ubiquitous to physiological activation? A ${ }^{1} \mathrm{H}$ functional MR spectroscopy study during motor activation in human brain at 7Tesla. Neuroimage 2014; 93(1): 138-145. DOI: 10.1016

13. Carandini M, Ferster D. A tonic hyperpolarization underlying contrast adaptation in cat visual cortex. Science 1997; 276(5314): 949-952.

14. Obata T, Liu TT, Miller KL, Luh WM, Wong EC, Frank LR, Buxton RB. Discrepancies between BOLD and flow dynamics in primary and supplementary motor areas: application of the balloon model to the interpretation of BOLD transients. Neuroimage 2004; 21(1): 144-153. 
15. Nakao Y, Itoh Y, Kuang TY, Cook M, Jehle J, Sokoloff L. Effects of anesthesia on functional activation of cerebral blood flow and metabolism. Proc. Natl. Acad. Sci. U. S. A. 2001; 98(13): 7593-7598.

16. Shulman RG, Hyder F, Rothman DL. Insights from neuroenergetics into the interpretation of functional neuroimaging: an alternative empirical model for studying the brain's support of behavior. J. Cereb. Blood Flow Metab. 2014; 34(11): 1721-1735. DOI: 10.1038

17. Kim T, Masamoto K, Fukuda M, Vazquez A, Kim SG. Frequency-dependent neural activity, CBF, and BOLD fMRI to somatosensory stimuli in isoflurane-anesthetized rats. Neuroimage 2010; 52(1): 224-233.

18. Garrett KM, Gan J. Enhancement of gamma-aminobutyric $\operatorname{acid}_{\mathrm{A}}$ receptor activity by alpha-chloralose. J. Pharmacol. Exp. Ther. 1998; 285(2): 680-686.

19. Hsu EW, Hedlund LW, MacFall JR. Functional MRI of the rat somatosensory cortex: effects of hyperventilation. Magn. Reson. Med. 1998; 40(3): 421-426.

20. Mégevand P, Troncoso E, Quairiaux C, Muller D, Michel CM, Kiss JZ. Long-term plasticity in mouse sensorimotor circuits after rhythmic whisker stimulation. J. Neurosci. 2009; 29(16): 5326-5335.

21. Sanganahalli BG, Herman P, Blumenfeld H, Hyder F. Oxidative neuroenergetics in event-related paradigms. J. Neurosci. 2009; 29 (6): 1707-1718.

22. Gruetter R, Tkác I. Field mapping without reference scan using asymmetric echo-planar techniques. Magn. Reson. Med. 2000; 43(2): 319-323.

23. Lei H, Mlynárik V, Just N, Gruetter R. Snapshot gradient-recalled echo-planar images of rat brains at long echo time at 9.4 T. Magn. Reson. Med. 1998; 26(7): 954-960.

24. Yan L, Zhuo Y, Ye Y, Xie SX, An J, Aguirre GK, Wang J. Physiological origin of low-frequency drift in blood oxygen level dependent (BOLD) functional magnetic resonance imaging (fMRI). Magn. Reson. Med. 2009; 61(4): 819-827.

25. Strupp JP. Stimulate a GUI based fMRI analysis software package. Neuroimage 1996; 3: S607.

26. Paxinos G, Watson C. The Rat Brain in Stereotaxic Coordinates. Academic: San Diego, CA, 1998.
27. Ances BM, Greenberg JH, Detre JA. Effects of variations in interstimulus interval on activation-flow coupling response and somatosensory evoked potentials with forepaw stimulation in the rat. J. Cereb. Blood Flow Metab. 2000; 20(2): 290-297.

28. Chung S, Li X, Nelson SB. Short-term depression at thalamocortical synapses contributes to rapid adaptation of cortical sensory responses in vivo. Neuron 2002; 34(3): 437-446.

29. Van Camp N, Verhoye M, Van der Linden A. Stimulation of the rat somatosensory cortex at different frequencies and pulse widths. NMR Biomed. 2006; 19(1): 10-17.

30. Kim YB, Kalthoff D, Po C, Wiedermann D, Hoehn M. Connectivity of thalamo-cortical pathway in rat brain: combined diffusion spectrum imaging and functional MRI at 11.7 T. NMR Biomed. 2012; 25(7): 943-952.

31. Quairiaux C, Armstrong-James M, Welker E. Modified sensory processing in the barrel cortex of the adult mouse after chronic whisker stimulation. J. Neurophysiol. 2007; 97(3): 2130-2147.

32. Cho YR, Pawela CP, Li R, Kao D, Schulte ML, Runquist ML, Yan JG, Matloub HS, Jaradeh SS, Hudetz AG, Hyde JS. Refining the sensory and motor ratunculus of the rat upper extremity using fMRI and direct nerve stimulation. Magn. Reson. Med. 2007; 58(5): 901-909.

33. Ogawa S, Tank DW, Menon R, Ellermann JM, Kim SG, Merkle $\mathrm{H}$, Ugurbil K. Intrinsic signal changes accompanying sensory stimulation: functional brain mapping with magnetic resonance imaging. Proc. Natl. Acad. Sci. U. S. A. 1992; 89(13): 5951-5955.

34. ladecola C, Yang G, Ebner TJ, Chen G. Local and propagated vascular responses evoked by focal synaptic activity in cerebellar cortex. J. Neurophysiol. 1997; 78(2): 651-659.

35. Aronoff R, Matyas F, Mateo C, Ciron C, Schneider B, Petersen CC. Long-range connectivity of mouse primary somatosensory barrel cortex. Eur. J. Neurosci. 2010; 31(12): 2221-2233.

36. Lowe AS, Beech JS, Williams SC. Small animal, whole brain fMRI: innocuous and nociceptive forepaw stimulation. Neuroimage 2007; 35(2): 719-728. 